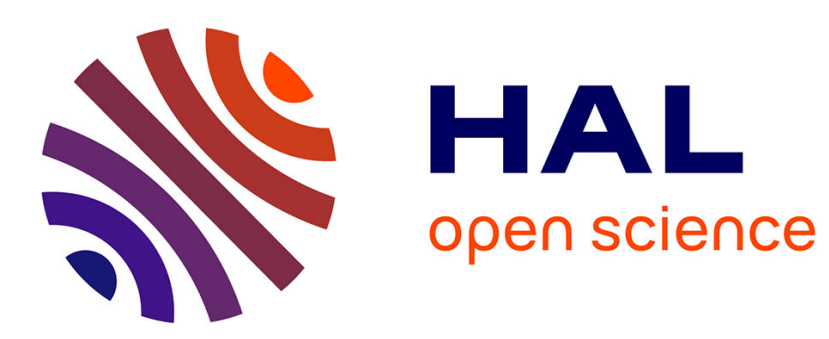

\title{
Almost all webs are not rank-perfect
}

Arnaud Pecher, Annegret K. Wagler

\section{To cite this version:}

Arnaud Pecher, Annegret K. Wagler. Almost all webs are not rank-perfect. Mathematical Programming, 2006, 105, pp.311-328. hal-00307763

\section{HAL Id: hal-00307763 https://hal.science/hal-00307763}

Submitted on 4 Nov 2008

HAL is a multi-disciplinary open access archive for the deposit and dissemination of scientific research documents, whether they are published or not. The documents may come from teaching and research institutions in France or abroad, or from public or private research centers.
L'archive ouverte pluridisciplinaire HAL, est destinée au dépôt et à la diffusion de documents scientifiques de niveau recherche, publiés ou non, émanant des établissements d'enseignement et de recherche français ou étrangers, des laboratoires publics ou privés. 
Mathematical Programming manuscript No.

(will be inserted by the editor)

Arnaud Pêcher ${ }^{1} \cdot$ Annegret K. Wagler ${ }^{2}$

\section{Almost all webs are not rank-perfect}

the date of receipt and acceptance should be inserted later

Abstract. Graphs with circular symmetry, called webs, are relevant w.r.t. describing the stable set polytopes of two larger graph classes, quasi-line graphs $[8,12]$ and claw-free graphs $[7,8]$. Providing a decent linear description of the stable set polytopes of claw-free graphs is a long-standing problem [9]. Ben Rebea conjectured a description for quasi-line graphs, see [12]; Chudnovsky and Seymour [2] verified this conjecture recently for quasi-line graphs not belonging to the subclass of fuzzy circular interval graphs and showed that rank facets are required in this case only. Fuzzy circular interval graphs contain all webs and even the problem of finding all facets of their stable set polytopes is open. So far, it is only known that stable set polytopes of webs with clique number $\leq 3$ have rank facets only $[5,16]$ while there are examples with clique number $\geq 4$ having non-rank facets $[10-12,14]$.

In this paper we prove, building on a construction for non-rank facets from [15], that the stable set polytopes of almost all webs with clique number $\geq 5$ admit non-rank facets. This adds support to the belief that these graphs are indeed the core of Ben Rebea's conjecture. Finally, we present a conjecture how to construct all facets of the stable set polytopes of webs.

Key words. web - rank-perfect graph - stable set polytope - (non-)rank facet

\section{Introduction}

Graphs with circular symmetry of their maximum cliques and stable sets are called webs: a web $W_{n}^{k}$ is a graph with vertices $1, \ldots, n$ where $i j$ is an edge if $i$ and $j$ differ by at $\operatorname{most} k(\bmod n)$ and $i \neq j$. The webs $W_{9}^{k}$ on nine vertices are depicted in Figure 1. Notice that webs are also called circulant graphs $C_{n}^{k}$ in [4] and that similar graphs $W(n, k)$ were introduced in [16].

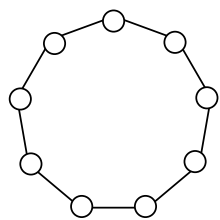

$W_{9}^{1}$

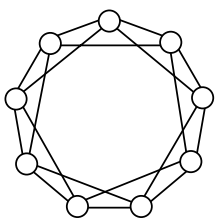

$W_{9}^{2}$

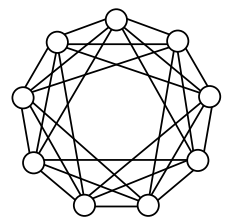

$W_{9}^{3}$

Fig. 1. The webs $W_{9}^{k}$ on nine vertices

Webs and line graphs belong to the classes of quasi-line graphs and claw-free graphs and are relevant w.r.t. describing the stable set polytopes of those larger graph classes [7, 8,12]. (The line graph of a graph $H$ is obtained by taking the edges of $H$ as nodes and connecting two nodes iff the corresponding edges of $H$ are incident. A graph is quasi-line

Arnaud Pêcher: Laboratoire Bordelais de Recherche Informatique (LaBRI)

351 cours de la Libération, 33405 Talence, France, e-mail: pecher a labri . fr

Annegret K. Wagler: Konrad-Zuse-Zentrum für Informationstechnik Berlin (ZIB)

Takustr. 7, 14195 Berlin, Germany, e-mail: wagler@zib. de

Correspondence to: wagler@zib.de 
(resp. claw-free) if the neighborhood of any node can be partitioned into two cliques (resp. does not contain any stable set of size 3$)$.)

The stable set polytope $\operatorname{STAB}(G)$ of $G$ is defined as the convex hull of the incidence vectors of all stable sets of the graph $G$. In order to describe $\operatorname{STAB}(G)$ by means of facetdefining inequalities, the "trivial" facets $x_{i} \geq 0$ for all vertices $i$ of $G$ and the clique constraints

$$
\sum_{i \in Q} x_{i} \leq 1
$$

for all cliques $Q \subseteq G$ are necessary. These two types of facets are sufficient to describe $\operatorname{STAB}(G)$ for perfect graphs $G$ only [3]. That are precisely the graphs without odd holes $W_{2 k+1}^{1}$ and odd antiholes $W_{2 k+1}^{k-1}$ as induced subgraphs.

A natural way to generalize clique constraints is to investigate rank constraints, that are $0 / 1$-constraints of the form

$$
\sum_{i \in G^{\prime}} x_{i} \leq \alpha\left(G^{\prime}\right)
$$

associated with arbitrary induced subgraphs $G^{\prime} \subseteq G$ where $\alpha\left(G^{\prime}\right)$ denotes the cardinality of a maximum stable set in $G^{\prime}$ (note $\alpha\left(G^{\prime}\right)=1$ holds iff $G^{\prime}$ is a clique). A graph is rankperfect if all non-trivial facets of its stable set polytope are rank constraints. The class of rank-perfect graphs contains all perfect graphs [3], odd holes and odd antiholes [13], line graphs [6], and the complements of webs [17].

A characterization of the rank facets in stable set polytopes of claw-free graphs was given by Galluccio and Sassano [7]. They showed that all rank facets can be constructed by means of standard operations from rank constraints associated with cliques, certain webs, and special line graphs. Finding all facets of their stable set polytopes is a long-standing problem (Grötschel, Lovász and Schrijver [9]), as claw-free graphs are not rank-perfect: Giles and Trotter [8], Oriolo [12], and Liebling et al. [11] found non-rank facets which occur even in the stable set polytopes of quasi-line graphs.

A famous conjecture due to Ben Rebea (see [12]) claims that the stable set polytopes of quasi-line graphs admit only one type of non-trivial facets, so-called clique family inequalities. Let $G=(V, E)$ be a graph, $\mathcal{F}$ be a family of (at least three inclusion-wise) maximal cliques of $G, p \leq|\mathcal{F}|$ be an integer, and define two sets as follows:

$$
\begin{aligned}
I(\mathcal{F}, p) & =\{i \in V:|\{Q \in \mathcal{F}: i \in Q\}| \geq p\} \\
O(\mathcal{F}, p) & =\{i \in V:|\{Q \in \mathcal{F}: i \in Q\}|=p-1\}
\end{aligned}
$$

The clique family inequality $(\mathcal{F}, p)$ is

$$
(p-r) \sum_{i \in I(\mathcal{F}, p)} x_{i}+(p-r-1) \sum_{i \in O(\mathcal{F}, p)} x_{i} \leq(p-r)\left\lfloor\frac{|\mathcal{F}|}{p}\right\rfloor
$$

with $r=|\mathcal{F}| \bmod p$ and $r>0$.

Oriolo [12] verified Ben Rebea's conjecture for line graphs and webs $W_{n}^{2}$ (note: the latter graphs are rank-perfect due to [5]). Chudnovsky and Seymour introduced recently the class of so-called fuzzy circular interval graphs and verified the conjecture for all quasi-line graphs which are not fuzzy circular interval graphs.

Let $\mathcal{C}$ be a circle and $\mathcal{I}=\left\{I_{1}, \ldots, I_{m}\right\}$ be a collection of intervals $I_{k}=\left[l_{k}, r_{k}\right]$ in $\mathcal{C}$ s.t. no interval in $\mathcal{I}$ is properly contained in another one and no two intervals in $\mathcal{I}$ share an endpoint. Moreover, let $V=\left\{v_{1}, \ldots, v_{n}\right\}$ be a finite multiset of points in $\mathcal{C}$ (i.e. $v_{i} \in \mathcal{C}$ may occur in $V$ with a multiplicity $>1$ ). The fuzzy circular interval graph $G(V, \mathcal{I})=\left(V, E_{1} \cup E_{2}\right)$ has node set $V$ and edge set $E_{1} \cup E_{2}$ where

$$
\begin{aligned}
& E_{1}=\left\{v_{i} v_{j}: \exists I_{k} \in \mathcal{I} \text { with } v_{i}, v_{j} \in I_{k} \text { and }\left\{v_{i}, v_{j}\right\} \neq\left\{l_{k}, r_{k}\right\}\right\} \\
& E_{2} \subseteq\left\{v_{i} v_{j}: \exists I_{k} \in \mathcal{I} \text { with } v_{i}=l_{k}, v_{j}=r_{k}\right\}
\end{aligned}
$$


(i.e., different endpoints of one interval are not necessarily joined by an edge).

Chudnovsky and Seymour recently proved that nonnegativity constraints, clique constraints, and rank constraints coming from clique family inequalities $(\mathcal{F}, 2)$ with $|\mathcal{F}|$ odd are the only necessary inequalities to describe stable set polytopes of quasi-line graphs which are not fuzzy circular interval graphs.

Webs are obviously quasi-line graphs as well as fuzzy circular interval graphs; the problem of describing their stable set polytopes is still open. So far, it is only known that webs $W_{n}^{1}$ are as holes perfect or rank-perfect [3,13]; the webs $W_{n}^{2}$ are rank-perfect by Dahl [5]. On the other hand, Kind [10] found (by means of the PORTA software ${ }^{1}$ ) examples of webs with clique number $>4$ which are not rank-perfect, e.g., $W_{31}^{4}, W_{25}^{5}, W_{29}^{6}, W_{33}^{7}, W_{28}^{8}, W_{31}^{9}$. Oriolo [12], Liebling et al. [11], and Pêcher and Wagler [14,15] presented further examples of such webs.

In this paper we prove, with the help of a construction for non-rank facets from [15], that there are only finitely many rank-perfect webs $W_{n}^{k}$ for all $k \geq 4$. Together with a result from [14] showing the same for the case $k=3$ we obtain that, for any $k \geq 3$, almost all webs $W_{n}^{k}$ are not rank-perfect. This adds support to the belief that webs as subclass of fuzzy circular interval graphs are the core of Ben Rebea's conjecture.

The paper is organized as follows: the next section describes as our main results the construction of infinite sequences of not rank-perfect webs and discusses consequences. The three following sections are devoted to the proofs of the three main theorems. We close with a conjecture which clique family inequalities give rise to facets in the stable set polytopes of webs.

\section{Main results}

For proving that almost all webs are not rank-perfect, we make use of a construction for non-rank facets from [15], introduced in the sequel. For that, we need the notion of proper weak non-rank facets. A facet $\mathbf{a}^{T} \mathbf{x} \leq c \alpha\left(G^{\prime}\right)$ of $\operatorname{STAB}(G)$ is a weak rank facet w.r.t. $G^{\prime} \subseteq$ $G$, if $a_{i}=c$ for every vertex $i$ of $G^{\prime}$ and if $G^{\prime}$ is rank facet-producing (i.e., $\sum_{i \in V\left(G^{\prime}\right)} x_{i} \leq$ $\alpha\left(G^{\prime}\right)$ defines a facet of $\operatorname{STAB}\left(G^{\prime}\right)$ ); any rank facet is a particular weak rank facet (with $a_{i}=c=1$ for every $i \in V\left(G^{\prime}\right)$ and $a_{i}=0$ otherwise). A weak rank facet is proper if $G^{\prime}$ is not a clique and non-rank if it cannot be scaled to have 0/1-coefficients only (i.e., it is not a rank-constraint).

Theorem 1. [15] If $\operatorname{STAB}\left(W_{n}^{k}\right)$ possesses a proper weak non-rank facet then also $\operatorname{STAB}\left(W_{n+k+1}^{k}\right)$ has a proper weak non-rank facet.

Therefore, if $\operatorname{STAB}\left(W_{n}^{k}\right)$ has a proper weak non-rank facet then all webs $W_{n+l(k+1)}^{k}$ $(l \geq 0)$ are not rank-perfect, too. Hence Theorem 1 implies the following corollary:

Corollary 1. [15] If there are $k+1$ webs $W_{n_{0}}^{k}, \ldots, W_{n_{k}}^{k}$ such that

$-\operatorname{STAB}\left(W_{n_{i}}^{k}\right)$ has a proper weak non-rank facet

$-n_{i}=i(\bmod k+1)$ for $0 \leq i \leq k$

then all webs $W_{n}^{k}$ with $n \geq \max \left\{n_{0}, \ldots, n_{k}\right\}-k$ are not rank-perfect.

For $k=3$, such a set $W_{33}^{3}, W_{42}^{3}, W_{51}^{3}, W_{60}^{3}$ is presented in [14], as consequence of the following theorem.

Theorem 2. [14] If $l=2(\bmod 3)$ and $l \geq 11$ then $\operatorname{STAB}\left(W_{3 l}^{3}\right)$ has a proper weak nonrankfacet.

\footnotetext{
${ }^{1}$ By PORTA it is possible to generate all facets of the convex hull of a given set of integer points, see http://www.zib.de
} 
Thus, Theorem 2 and Corollary 1 imply that all webs $W_{n}^{3}$ with $n>56$ are not rankperfect. The aim of this paper is to provide such a set $W_{n_{0}}^{k}, \ldots, W_{n_{k}}^{k}$ of not rank-perfect webs for each value $k \geq 4$. For that, we consider special clique family inequalities giving rise to proper weak non-rank facets. A clique family inequality $(\mathcal{Q}, p)$ is associated with a proper subweb $W_{n^{\prime}}^{k^{\prime}}$ of a web $W_{n}^{k}$ if $\mathcal{Q}=\left\{Q_{i}: i \in W_{n^{\prime}}^{k^{\prime}}\right\}$ is chosen as clique family and $p=k^{\prime}+1$, where $Q_{i}=\{i, \ldots, i+k\}$ denotes the maximum clique of $W_{n}^{k}$ starting in vertex $i$. Note that the clique number of a web $W_{n}^{k}$ is $k+1$ and the stability number is $\left\lfloor\frac{n}{k+1}\right\rfloor$.

Lemma 1. [14] Let $W_{n^{\prime}}^{k^{\prime}} \subset W_{n}^{k}$ be a proper induced subweb. The clique family inequality $\left(\mathcal{Q}, k^{\prime}+1\right)$ associated with $W_{n^{\prime}}^{k^{\prime}}$

$$
\left(k^{\prime}+1-r\right) \sum_{i \in I\left(\mathcal{Q}, k^{\prime}+1\right)} x_{i}+\left(k^{\prime}-r\right) \sum_{i \in O\left(\mathcal{Q}, k^{\prime}+1\right)} x_{i} \leq\left(k^{\prime}+1-r\right) \alpha\left(W_{n^{\prime}}^{k^{\prime}}\right)
$$

where $r=n^{\prime} \bmod \left(k^{\prime}+1\right), 0<r<k^{\prime}+1$, is a valid inequality for $\operatorname{STAB}\left(W_{n}^{k}\right)$, such that $W_{n^{\prime}}^{k^{\prime}} \subseteq I(\mathcal{F}, p)$ holds.

For illustration, look at the smallest not rank-perfect web $W_{25}^{5}$. Its non-rank facets are clique family inequalities associated with induced subwebs $W_{10}^{2} \subseteq W_{25}^{5}$ (note that the vertex sets 1, 2, 6, 7, 11, 12, 16, 17, 21, 22 and 1, 3, 6, 8, 11, 13, 16, 18, 21, 23 both induce a $W_{10}^{2} \subseteq W_{25}^{5}$, see the black vertices in Figure 2).
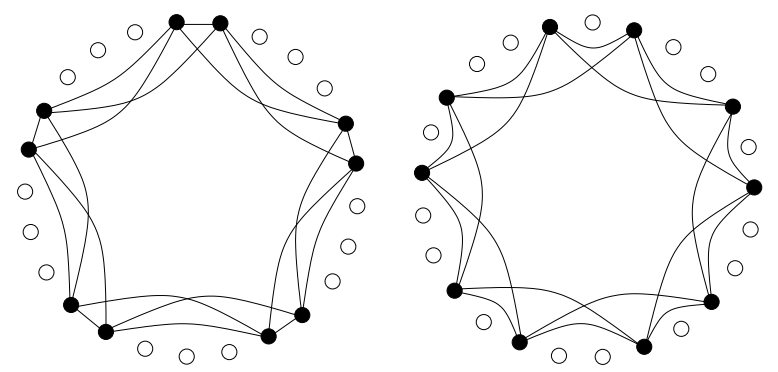

Fig. 2. : The induced subwebs $W_{10}^{2} \subseteq W_{25}^{5}$

Choosing $\mathcal{Q}=\left\{Q_{i}: i \in W_{10}^{2}\right\}$ yields $p=\omega\left(W_{10}^{2}\right)=3$ in both cases. All remaining vertices are covered 2 times, hence $O(\mathcal{Q}, p)=W_{25}^{5}-W_{10}^{2}$ follows. The corresponding clique family inequality $(\mathcal{Q}, 3)$ is

$$
2 \sum_{i \in W_{10}^{2}} x_{i}+1 \sum_{i \notin W_{10}^{2}} x_{i} \leq 2 \alpha\left(W_{10}^{2}\right)
$$

due to $r=|\mathcal{Q}| \bmod p=1$ and yields a non-rank facet of $\operatorname{STAB}\left(W_{25}^{5}\right)$.

The main results of this paper prove that several clique family inequalities $\left(\mathcal{Q}, k^{\prime}+1\right)$ associated with different regular subwebs $W_{n^{\prime}}^{k^{\prime}}$ induce proper weak non-rank facets (note that $\left(\mathcal{Q}, k^{\prime}+1\right)$ is a proper weak non-rank constraint if $\left.r<k^{\prime}\right)$. A subweb $W_{n^{\prime}}^{k^{\prime}} \subset W_{n}^{k}$ is called $\left(b_{1}, w_{1}, \ldots, b_{t}, w_{t}\right)$-regular, if the vertices of $W_{n^{\prime}}^{k^{\prime}}$ occur in $W_{n}^{k}$ in equal blocks where $b_{i}$ consecutive vertices from $W_{n^{\prime}}^{k^{\prime}}$ alternate with $w_{i}$ consecutive vertices outside $W_{n^{\prime}}^{k^{\prime}}$, for $1 \leq i \leq t$. The two subwebs $W_{10}^{2} \subseteq W_{25}^{5}$ presented in Figure 2 show a (2,3)-regular and a $(1,1,1,2)$-regular subweb, resp. In Section 3, we show the following:

Theorem 3. For any $k \geq 5$, consider a $\left(k^{\prime}, k-k^{\prime}\right)$-regular subweb $W_{l k^{\prime}}^{k^{\prime}} \subset W_{l k}^{k}$ with $2 \leq k^{\prime} \leq k-3$ and odd $l \geq 3$. The clique family inequality

$$
2 \sum_{i \in W_{l k^{\prime}}^{k^{\prime}}} x_{i}+1 \sum_{i \notin W_{l k^{\prime}}^{k^{\prime}}} x_{i} \leq 2 \alpha\left(W_{l k^{\prime}}^{k^{\prime}}\right)
$$


associated with $W_{l k^{\prime}}^{k^{\prime}}$ is a proper weak non-rank facet of $\operatorname{STAB}\left(W_{l k}^{k}\right)$ if $l=2\left(\bmod k^{\prime}+1\right)$ and $\alpha\left(W_{l k^{\prime}}^{k^{\prime}}\right)<\alpha\left(W_{l k}^{k}\right)$.

As a consequence, we obtain many different infinite sequences of not rank-perfect webs, among them the required base sets for all even values of $k \geq 6$ (but not for the odd values $k \geq 5$ since all webs in the latter sequences have an odd number of vertices). For any even $k \geq 6$, choosing $k^{\prime}=\frac{k}{2}$ if $k=0(\bmod 4)$ and $k^{\prime}=\frac{k}{2}-1$ if $k=2$ $(\bmod 4)$ and $l=\left(k^{\prime}+3\right)+\left(k^{\prime}+1\right) 2 j$ for $j \geq 1$ in both cases as odd values of $l$ with $l=2\left(\bmod k^{\prime}+1\right)$ satisfies the precondition of Theorem 3 . Thus, we obtain the following infinite sequences of not rank-perfect webs:

Theorem 4. Let $k \geq 6$ be even. Then for every integer $j \geq 1$ holds

$-\operatorname{STAB}\left(W_{\left(\frac{k+6}{2}+(k+2) j\right) k}^{k}\right)$ has a proper weak non-rank facet if $k=0(\bmod 4)$;

$-\operatorname{STAB}\left(W_{\left(\frac{k+4}{2}+k j\right) k}^{k}\right)$ has a proper weak non-rank facet if $k=2(\bmod 4)$.

That means for, e.g., $k=6$ that there is an infinite sequence $W_{66}^{6}, W_{102}^{6}, W_{138}^{6}, W_{174}^{6}$, $W_{210}^{6}, W_{246}^{6}, W_{282}^{6}, \ldots$ of not rank-perfect webs. Corollary 1 implies, therefore, that all webs $W_{n}^{6}$ with $n \geq 276$ are not rank-perfect. More generally, for every $1 \leq j \leq k+1$, we have

$$
\left(\frac{k+6}{2}+(k+2) j\right) k=-\frac{k+6}{2}-j \quad(\bmod k+1)
$$

and

$$
\left(\frac{k+4}{2}+k j\right) k=-\frac{k+4}{2}+j \quad(\bmod k+1)
$$

thus, the sequences contain the required base sets. Furthermore, if $k \geq 6$ then

$$
\left(\frac{k+6}{2}+(k+2)(k+1)\right) k<2(k+1)^{3}
$$

follows and Theorem 4 and Corollary 1 imply together:

Corollary 2. For any even $k \geq 6$, all webs $W_{n}^{k}$ with $n \geq 2(k+1)^{3}$ are not rank-perfect.

It remains to construct the required base sets for $k=4$ and all odd values of $k \geq 5$. The case $k=4$ is treated in Section 4 by constructing sequences of clique family inequalities associated with regular subwebs $W_{l}^{2} \subset W_{2 l}^{4}$ :

Theorem 5. The clique family inequality $(\mathcal{Q}, 3)$

$$
2 \sum_{i \in W_{l}^{2}} x_{i}+1 \sum_{i \notin W_{l}^{2}} x_{i} \leq 2 \alpha\left(W_{l}^{2}\right)
$$

associated with a $(1,1)$-regular subweb $W_{l}^{2} \subset W_{2 l}^{4}$ is a proper weak non-rank facet of $\operatorname{STAB}\left(W_{2 l}^{4}\right)$ if $l=1(\bmod 3)$ and $l \geq 13$.

Due to Theorem 5, the stable set polytopes of the webs $W_{26}^{4}, W_{32}^{4}, W_{38}^{4}, W_{44}^{4}$, and $W_{50}^{4}$ have a proper weak non-rank facet. Hence Corollary 1 implies that all webs $W_{n}^{4}$ with $n>45$ are not rank-perfect.

For each odd $k \geq 5$, we extend the result for $k=3$ from [14] by considering the clique family inequality associated with the $(k-1,1)$-regular subweb $W_{l(k-1)}^{k-1} \subset W_{l k}^{k}$ : 
Theorem 6. The clique family inequality $(\mathcal{Q}, k)$

$$
2 \sum_{i \in W_{l(k-1)}^{k-1}} x_{i}+1 \sum_{i \notin W_{l(k-1)}^{k-1}} x_{i} \leq 2 \alpha\left(W_{l(k-1)}^{k-1}\right)
$$

associated with a $(k-1,1)$-regular subweb $W_{l(k-1)}^{k-1}$ is a proper weak non-rank facet of $\operatorname{STAB}\left(W_{l k}^{k}\right)$ for any odd $k \geq 5$ if $l=3 k+2$.

The sequence of the $k+1$ webs $W_{l^{\prime} k+2}^{k}$ with $3 \leq k^{\prime} \leq 3+k$ webs is the required base set for any odd $k \geq 5$, as

$$
k\left(l^{\prime} k+2\right) \bmod (k+1)=\left(l^{\prime}-2\right) \bmod (k+1)
$$

Thus, Theorem 6 and Corollary 1 imply together:

Corollary 3. $W_{n}^{k}$ with $n \geq((k+3) k+1) k$ is not rank-perfect for any odd $k \geq 5$.

In summary, all the above results show:

Corollary 4. A web $W_{n}^{k}$ is not rank-perfect if

$-k=3$ and $n \geq 57$,

$-k=4$ and $n \geq 46$,

$-k \geq 5$ is odd and $n \geq((3+k) k+1) k$,

$-k \geq 6$ is even and $n \geq 2(k+1)^{3}$.

Thus, for any $k \geq 3$ there are only finitely many rank-perfect webs $W_{n}^{k}$ implying:

Corollary 5. Almost all webs with given clique size at least 4 are not rank-perfect.

The following three sections contain the proofs of the main results Theorem 3, Theorem 5, and Theorem 6.

\section{Proof of Theorem 3}

For any $k \geq 5$, let $W_{l k^{\prime}}^{k^{\prime}}$ be a $\left(k^{\prime}, k-k^{\prime}\right)$-regular subweb of $W_{l k}^{k}$ with $2 \leq k^{\prime} \leq k-3$ and odd $l \geq 3$. By assumption, we have $l=2\left(\bmod k^{\prime}+1\right)$ and $\alpha\left(W_{l k^{\prime}}^{k^{\prime}}\right)<\alpha\left(W_{l k}^{k}\right)$.

In order to prove Theorem 3 , we have to establish that the inequality (3)

$$
2 \sum_{i \in W_{l k^{\prime}}^{k^{\prime}}} x_{i}+1 \sum_{i \notin W_{l k^{\prime}}^{k^{\prime}}} x_{i} \leq 2 \alpha\left(W_{l k^{\prime}}^{k^{\prime}}\right)
$$

is valid and facet-inducing for $\operatorname{STAB}\left(W_{l k}^{k}\right)$.

Validity follows from Lemma 1 : since $l=2\left(\bmod k^{\prime}+1\right)$, we have $l k^{\prime}=-2$ $\left(\bmod k^{\prime}+1\right)$ and therefore the remainder $r$ of the division of $l k^{\prime}$ by $k^{\prime}+1$ is equal to $k^{\prime}-1$. Therefore the valid inequality (2) associated with the subweb $W_{l k^{\prime}}^{k^{\prime}}$ is

$$
2 \sum_{i \in I\left(\mathcal{Q}, k^{\prime}+1\right)} x_{i}+\sum_{i \in O\left(\mathcal{Q}, k^{\prime}+1\right)} x_{i} \leq 2 \alpha\left(W_{l k^{\prime}}^{k^{\prime}}\right)
$$

where $W_{l k^{\prime}}^{k^{\prime}} \subseteq I(\mathcal{F}, p)$ holds. Therefore, inequality (3) is a valid inequality.

To prove that inequality (3) is facet-inducing, we may define the set of vertices $V^{\prime}$ of the $\left(k^{\prime}, k-k^{\prime}\right)$-regular subweb $W_{l k^{\prime}}^{k^{\prime}}$ w.l.o.g. as

$$
V^{\prime}=\bigcup_{0 \leq j<l}\left\{k \cdot j+1, k \cdot j+2, \ldots, k \cdot j+k^{\prime}\right\}
$$


(where $l \geq 5$ and $l=2\left(\bmod k^{\prime}+1\right)$ )

For convenience, we call the vertices in $V^{\prime}$ black vertices and all remaining vertices white vertices. A black set is a set of black vertices and likewise a white set is a set of white vertices.

The following lemma from [15] is essential for the proof. It provides a characterization when a valid inequality $\mathbf{a}^{T} \mathbf{x} \leq b$ is a facet of the stable set polytope of a general graph $G$. For that we need the following notions. A root of $\mathbf{a}^{T} \mathbf{x} \leq b$ is any stable set of $G$ satisfying the inequality at equality. A pair $i, j$ of vertices is a-critical in $G$ if there are two roots $S_{1}$ and $S_{2}$ of $\mathbf{a}^{T} \mathbf{x} \leq b$ such that $\{i\}=S_{1} \backslash S_{2}$ and $\{j\}=S_{2} \backslash S_{1}$. A subset $V^{\prime}$ of $V(G)$ is a-connected if the graph with vertex set $V^{\prime}$ and edge set $\left\{i j \mid i, j \in V^{\prime}, i j\right.$ a-critical in $\left.G\right\}$ is connected.

All matrices in this paper have rational entries (in fact integer entries). If $M$ is any square matrix, then $|M|$ stands for the determinant of $M$.

Lemma 2. [15] Let $\mathbf{a}^{T} \mathbf{x} \leq b$ be a valid inequality for $\operatorname{STAB}(G)$ with $b \neq 0$. Consider a partition $V_{1}, \ldots, V_{p}$ of $V(G)$ such that $V_{i}$ is a-connected for every $1 \leq i \leq p$. The inequality $\mathbf{a}^{T} \mathbf{x} \leq b$ is facet-defining if and only if there are $p$ roots $S_{1}, \ldots, S_{p}$ with

$$
\left|\begin{array}{ccc}
\left|S_{1} \cap V_{1}\right| & \cdots & \left|S_{1} \cap V_{p}\right| \\
\vdots & \vdots \\
\left|S_{p} \cap V_{1}\right| & \cdots & \left|S_{p} \cap V_{p}\right|
\end{array}\right| \neq 0
$$

If the involved inequality $\mathbf{a}^{T} \mathbf{x} \leq b$ is the full rank-constraint $\mathbf{1}^{T} \mathbf{x} \leq \alpha$, we use the terms $\alpha$-critical and $\alpha$-connected instead of a-critical and a-connected respectively.

Notice that Chvátal [3] called $\alpha$-critical edges simply "critical" and that Lemma 2 generalizes the well-known result of Chvátal [3] that a graph $G$ is rank facet-producing if the set of its critical edges induces a conncted subgraph of $G$.

We now proceed to the proof that inequality (3) is facet-inducing,

Claim. The black set $V^{\prime}$ is a-connected w.r.t. the valid inequality (3).

\section{Proof.}

If $l k^{\prime}=0\left(\bmod k^{\prime}+1\right)$ then $-l=0\left(\bmod k^{\prime}+1\right)$ and therefore $l=0\left(\bmod k^{\prime}+1\right)$, in contradiction with $l=2\left(\bmod k^{\prime}+1\right)$, as $k^{\prime} \geq 2$. Hence $k^{\prime}+1$ is not a divisor of $l k^{\prime}$. Hence we have $l k^{\prime}=\alpha\left(G\left[V^{\prime}\right]\right)\left(k^{\prime}+1\right)+r$ with $1 \leq r \leq k^{\prime}$. Let $S_{1}=\left\{1,2+\left(k^{\prime}+1\right), 2+\right.$ $\left.2\left(k^{\prime}+1\right), \ldots, 2+\left(\alpha\left(G\left[V^{\prime}\right]\right)-1\right)\left(k^{\prime}+1\right)\right\}$ and $S_{2}=\left\{2,2+\left(k^{\prime}+1\right), 2+2\left(k^{\prime}+1\right), \ldots, 2+\right.$ $\left.\left(\alpha\left(G\left[V^{\prime}\right]\right)-1\right)\left(k^{\prime}+1\right)\right\}$. Since $2+\left(\alpha\left(G\left[V^{\prime}\right]\right)-1\right)\left(k^{\prime}+1\right)=2+\left(l k^{\prime}-r\right)-\left(k^{\prime}+1\right) \leq l k^{\prime}-k^{\prime}$, $S_{1}$ and $S_{2}$ are both maximum stable sets of $G\left[V^{\prime}\right]$. Hence, the edge $\{1,2\}$ of $G\left[V^{\prime}\right]$ is $\alpha$-critical. By circular symmetry of $G\left[V^{\prime}\right]$, this implies that $G\left[V^{\prime}\right]$ is $\alpha$-connected. Since $\alpha\left(G\left[V^{\prime}\right]\right)=\alpha^{\prime}$, this implies that $V^{\prime}$ is a-connected.

Claim. We have $l k^{\prime}>\left(\alpha^{\prime}-2\right)\left(k^{\prime}+1\right)+3 k^{\prime}$.

Proof. Since $l=2\left(\bmod k^{\prime}+1\right)$, we have $l k^{\prime}=k^{\prime}-1\left(\bmod k^{\prime}+1\right)$. Hence $l k^{\prime}-$ $\alpha^{\prime}\left(k^{\prime}+1\right)=k^{\prime}-1$. It follows that $l k^{\prime}-\alpha^{\prime}\left(k^{\prime}+1\right)>3 k^{\prime}-2\left(k^{\prime}+1\right)$. Thus $l k^{\prime}>$ $\left(\alpha^{\prime}-2\right)\left(k^{\prime}+1\right)+3 k^{\prime}$.

Claim. We obtain $\alpha\left(W_{l k^{\prime}}^{k^{\prime}} \backslash\left[1,3 k^{\prime}\right]\right) \geq \alpha\left(W_{l k^{\prime}}^{k^{\prime}}\right)-1$.

Proof. By the previous Claim, the set $S^{\prime}:=\left\{3 k^{\prime}+1,3 k^{\prime}+\left(k^{\prime}+1\right)+1, \ldots, 3 k^{\prime}+\left(\alpha^{\prime}-\right.\right.$ $\left.2)\left(k^{\prime}+1\right)+1\right\}$ is a stable set of size $\alpha^{\prime}-1$ of $W_{l k^{\prime}}^{k^{\prime}} \backslash\left[1,3 k^{\prime}\right]$ and the result follows.

Claim. For every $0 \leq i<l$, the white set $V_{i}:=i k+\left\{k^{\prime}+1, \ldots, k\right\}$ is a-connected. 


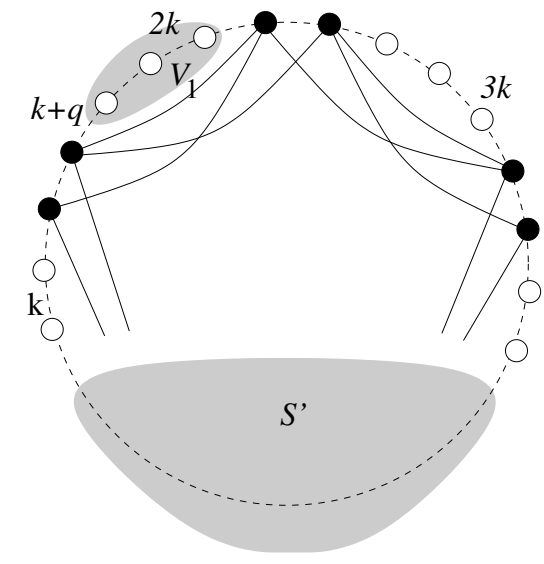

(a)

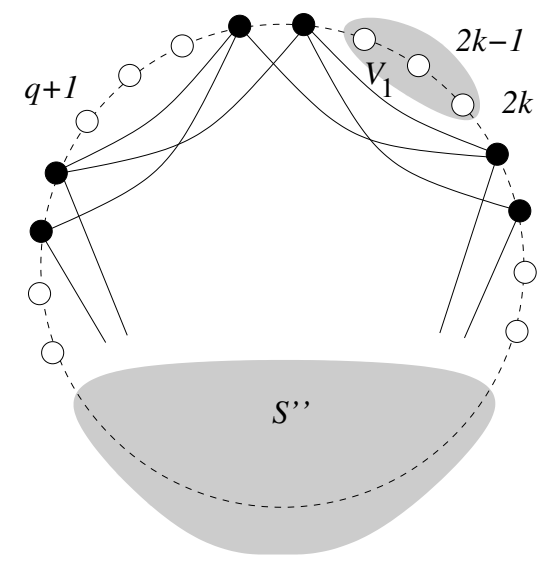

(b)

Fig. 3. : The roots for the proof of the fourth Claim with $k=5$ and $k^{\prime}=2:$ (a) the roots $S_{j}=S^{\prime} \cup\{3 k, j\}$ (b) the stable sets $S_{1}=S^{\prime \prime} \cup\left\{k^{\prime}+1,2 k-1\right\}$ and $S_{2}=S^{\prime \prime} \cup\left\{k^{\prime}+1,2 k\right\}$

Proof. We are going to prove that $V_{1}$ is a-connected. By the previous Claim, there is a black stable set $S^{\prime}$ of size $\alpha^{\prime}-1$ in $G \backslash[k+1,4 k]$. For every $k+k^{\prime}+1 \leq j \leq 2 k-1$, the set $S_{j}:=S^{\prime} \cup\{3 k, j\}$ is obviously a root of (3), hence the edges $\left\{k^{\prime}+\bar{k}+1, k^{\prime}+k+\right.$ $2\}, \ldots,\{2 k-2,2 k-1\}$, are a-critical (see Fig. 3(a)).

It remains to show that the edge $\{2 k-1,2 k\}$ is a-critical. By the previous Claim again, there exists a black stable set $S^{\prime \prime}$ of size $\alpha^{\prime}-1$ in $G \backslash\left[k^{\prime}-k+1,3 k\right]$. The set $S_{1}:=S^{\prime \prime} \cup\left\{k^{\prime}+1\right\} \cup\{2 k-1\}$ is a root as $k^{\prime}+1+k<2 k-1$ (since $k^{\prime} \leq k-3$ ). The set $S_{2}:=S^{\prime \prime} \cup\left\{k^{\prime}+1\right\} \cup\{2 k\}$ is also a root (see Fig. 3(b)). Hence $\{2 k-1,2 k\}$ is a-critical and, therefore, $V_{1}$ is a-connected.

Likewise, the sets $V_{0}, V_{2} \ldots, V_{l-1}$ are a-connected.

Claim. For every $0 \leq i<l$ there exists a stable set $S_{i}$ such that $S_{i}$ meets $V^{\prime}$ in exactly $\alpha^{\prime}-1$ vertices, $V_{i}$ in exactly one vertex, and $V_{i+1}$ in also exactly one vertex.

Proof. For every $0 \leq i<l$, there exists a black stable set $S_{i}^{\prime}$ of size $\alpha^{\prime}-1$ in $G \backslash[i k+$ $1,(i+3) k]$. Let $S_{i}$ be the stable set $S_{i}^{\prime} \cup\left\{i k+k^{\prime}+1\right\} \cup\left\{(i+1) k+k^{\prime}+2\right\}$. Then $S_{0}, \ldots, S_{l-1}$ give the result.

Let $S^{\prime}$ be a maximum stable set of $G\left[V^{\prime}\right]$.

Hence we have

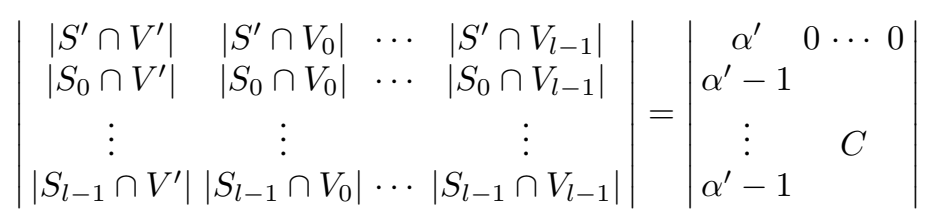

where $C$ is the $(2, l)$-circulant matrix with top row $(1,1,0, \ldots, 0)$ of size $l$. The matrix $C$ is invertible as $l$ is odd. Hence the above determinant is non-zero.

Therefore, the proof of Theorem 3 is done due to Lemma 2.

\section{Proof of Theorem 5}

In order to obtain an infinite sequence of not rank-perfect webs $W_{n}^{4}$ we consider, for any even $n=2 l$, the (1,1)-regular subweb $W_{l}^{2} \subset W_{2 l}^{4}$. In the remaining part of this section, let $V=\{1, \ldots, 2 l\}$ denote the vertex set of $W_{2 l}^{4}$ and 


$$
\begin{aligned}
& V_{o}=\{1,3, \ldots, 2 l-1\} \\
& V_{e}=\{2,4, \ldots, 2 l\}
\end{aligned}
$$

denote the subsets of vertices with odd resp. even index in $V$. Then both sets $V_{o}$ and $V_{e}$ induce a subweb $W_{l}^{2}$ of $W_{2 l}^{4}$, see Figure 4(a).

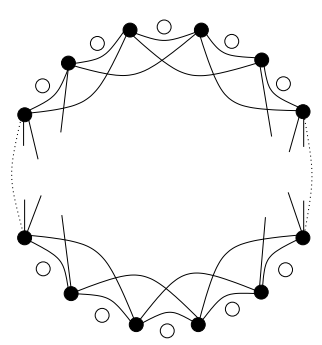

(a)

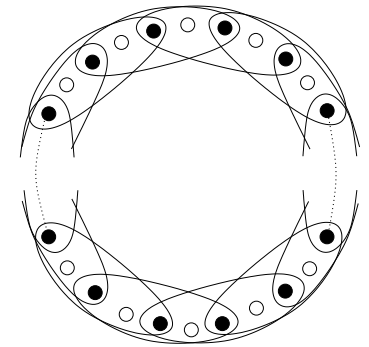

(b)

Fig. 4. The partition of $W_{2 l}^{4}$

In the sequel, consider the clique family inequality $(\mathcal{Q}, 3)$ of $\operatorname{STAB}\left(W_{2 l}^{4}\right)$ associated with the subweb $W_{l}^{2}$ induced by $V_{o}$. This means, we choose $\mathcal{Q}=\left\{Q_{i}: i \in V_{o}\right\}$ (the cliques of $\mathcal{Q}$ are represented in Figure 4(b) where the vertices from $V_{o}$ are drawn in black) and obtain $I(\mathcal{Q}, 3)=V_{o}$ and $O(\mathcal{Q}, 3)=V_{e}$. The clique family inequality $(\mathcal{Q}, 3)$ associated with $W_{l}^{2}=W_{2 l}^{4}\left[V_{o}\right]$ is, therefore,

$$
(3-r) \sum_{i \in V_{o}} x_{i}+(2-r) \sum_{i \in V_{e}} x_{i} \leq(3-r) \alpha\left(W_{l}^{2}\right)
$$

and it is a non-rank constraint if $r=1$. Hence, $(\mathcal{Q}, 3)$ corresponds to the studied inequality (4) if $l=1(\bmod 3)$. We prove that it is a facet for all $l \in\{13,16,19, \ldots\}$.

For that, we have to present $2 l$ roots of (4) whose incidence vectors are linearly independent. (Recall that a root of (4) is a stable set of $W_{2 l}^{4}$ satisfying (4) at equality.) It follows from [16] that a web $W_{n}^{k}$ produces the full rank facet $\sum_{i \in W_{n}^{k}} x_{i} \leq \alpha\left(W_{n}^{k}\right)$ if and only if $(k+1) \backslash n$. Thus $W_{l}^{2}$ is facet-producing as $l=1(\bmod 3)$ and the maximum stable sets of $W_{l}^{2}$ yield, therefore, already $l$ independent roots of (4). We need a set $\mathcal{S}$ of further $l$ roots of (4) admitting vertices from $V_{o}$ as well as from $V_{e}$, called mixed roots, and are independent, too.

We construct, for all $l \geq 13$ with $l=1(\bmod 3)$, a set $\mathcal{S}$ of $l$ mixed roots $S$ of size $\alpha_{o}+1$ with $\left|S \cap V_{o}\right|=\alpha_{o}-1$ and $\left|S \cap V_{e}\right|=2$ where $\alpha_{o}=\alpha\left(W_{l}^{2}\right)=\left\lfloor\frac{l}{3}\right\rfloor$ (notice that $2\left(\alpha_{o}-1\right)+2=2 \alpha_{o}$ according to the coefficients of (4)).

For that, we use the following representation of stable sets $S \subseteq W_{2 l}^{4}$ of size $\alpha_{o}+1$ : choose a start vertex $i \in S$ and the distance vector $D=\left(d_{1}, \ldots, d_{\alpha_{o}+1}\right)$ containing the distances between two consecutive vertices of $S$, i.e.,

$$
S=S(i, D)=\left\{i, i+d_{1},\left(i+d_{1}\right)+d_{2}, \ldots,\left(i+\sum_{j<\alpha_{o}} d_{j}\right)+d_{\alpha_{o}}\right\}
$$

where

$$
\sum_{j \leq \alpha_{o}+1} d_{j}=2 l \text { (i.e. } i=i+\sum_{j \leq \alpha_{o}+1} d_{j} \bmod 2 l \text { ) }
$$

and $d_{j}>k=4$ for $1 \leq j \leq \alpha_{o}+1$ (ensuring that $S$ is a stable set in $W_{2 l}^{4}$ ).

Claim. Let $D=\left(d_{1}, \ldots, d_{\alpha_{o}+1}\right)$ be a distance vector such that $D$ has 4 entries $d_{j}$ equal to 5 and $\alpha_{o}-3$ entries $d_{j}$ equal to 6 . Then $S(i, D)$ is a stable set of $W_{2 l}^{4}$ for every vertex $i$ for all $l=1 \bmod 3$. 
Proof. We have to show that $\sum_{d_{j} \in D} d_{j}=2 l$ holds. Recalling $\alpha_{o}=\left\lfloor\frac{l}{3}\right\rfloor$ and $l=1$ $(\bmod 3)$, we obtain

$$
5 \cdot 4+6\left(\alpha_{o}-3\right)=2+6 \alpha_{o}=2+6\left\lfloor\frac{l}{3}\right\rfloor=2+6 \frac{l-1}{3}=2+2(l-1)=2 l
$$

as required.

We define two different distance vectors with $\alpha_{o}+1$ entries each by

$$
\begin{aligned}
& D_{1}=(5,5,5,6, \ldots, 6,5) \\
& D_{2}=(5,6,5,5,6, \ldots, 6,5)
\end{aligned}
$$

and show that they produce the studied mixed roots of (4).

Claim. For every $i \in V_{e}, S\left(i, D_{1}\right)\left(\right.$ resp. $\left.S\left(i, D_{2}\right)\right)$ contains precisely the 2 vertices $i, i+10$ (resp. $i, i+16$ ) from $V_{e}$ and $\alpha_{o}-1$ vertices from $V_{o}$.

Proof. By the choice of $D_{1}$ and $D_{2}$ and the previous claim, both stable sets have size $\alpha_{o}+1$ and start with a vertex in $V_{e}$ (due to $i \in V_{e}$ ). The parity of the distances $d_{j}$ in $D_{1}$ and $D_{2}$ implies that the third vertex $i+10$ of $S\left(i, D_{1}\right)$ and the fourth vertex $i+16$ of $S\left(i, D_{2}\right)$ is in $V_{e}$ again, whereas all remaining $\alpha_{o}-1$ vertices belong to $V_{o}$ (see Figure 5 , vertices in $V_{e}\left(\right.$ resp. $\left.V_{o}\right)$ are drawn in white (resp. black)).
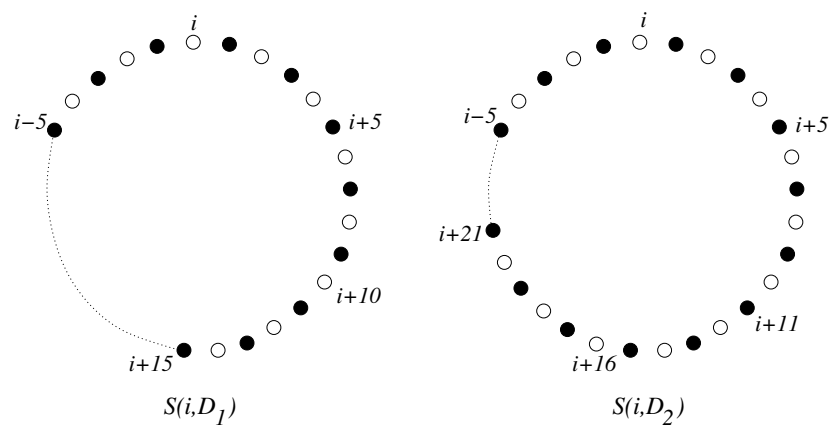

Fig. 5. The mixed roots $S\left(i, D_{1}\right)$ and $S\left(i, D_{2}\right)$

Hence, each set $S\left(i, D_{1}\right)$ and $S\left(i, D_{2}\right)$ with $i \in V_{e}$ is a mixed root of (4). We now have to choose a set $\mathcal{S}$ of $l$ distinct mixed roots of (4) with linearly independent incidence vectors.

Assume that $\mathcal{S}$ is such a set and denote by $A_{\mathcal{S}}$ the square matrix containing the incidence vectors of $l$ linearly independent maximum stable sets of $W_{l}^{2}=W_{2 l}^{4}\left[V_{o}\right]$ as first $l$ rows and the incidence vectors of the $l$ mixed roots in $\mathcal{S}$ as last $l$ rows. Order the columns of $A_{\mathcal{S}}$ s.t. the first (resp. last) $l$ columns correspond to the vertices in $V_{o}$ (resp. $V_{e}$ ), both in increasing order. Then $A_{\mathcal{S}}$ has the block structure

$$
A_{\mathcal{S}}=\left(\begin{array}{c|c}
A_{11} & 0 \\
\hline A_{21} & A_{22}
\end{array}\right)
$$

where the $l \times l$-matrix $A_{11}$ is invertible since $W_{l}^{2}$ is facet-producing by [16] (in the considered case with $l=1(\bmod 3))$.

In the sequel, we provide a set $\mathcal{S}$ of $l$ distinct mixed roots s.t. $A_{22}$ (i.e. the intersection of the mixed roots with $V_{e}$ ) is an invertible $l \times l$-matrix (then $A_{\mathcal{S}}$ is invertible due to its block structure).

Claim. For every $l \geq 13$, there is a set $\mathcal{S}$ of $l$ mixed roots of (4) containing precisely 2 vertices of $V_{e}$ s.t. the $l \times l$-submatrix $A_{22}$ of $A_{\mathcal{S}}$ is invertible. 


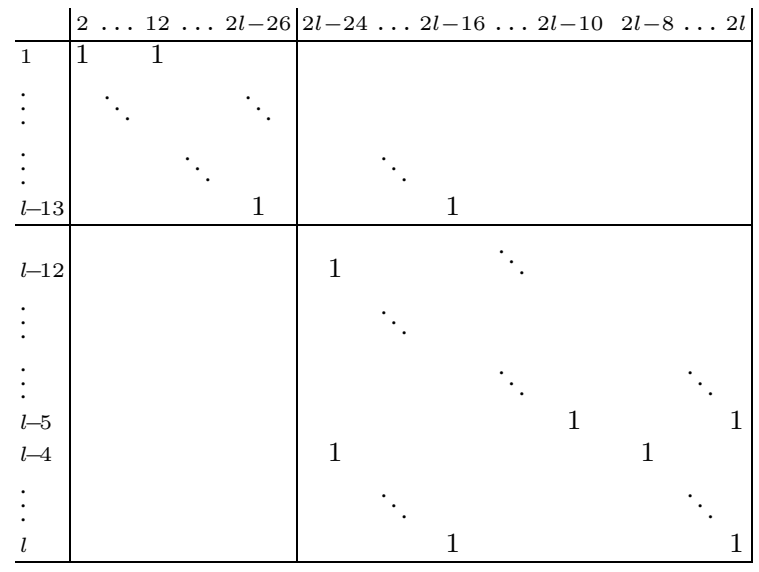

Fig. 6. The $l \times l$-matrix $A_{22}$

Proof. For any $i \in V_{e}$, both $S\left(i, D_{1}\right)$ and $S\left(i, D_{2}\right)$ are roots of (4) by the previous claim. Chose $S\left(i, D_{1}\right)$ with $i \in\{2,4, \ldots, 2 l-10\}$ as the first $l-5$ roots in $\mathcal{S}$ and $S\left(i, D_{2}\right)$ with $i \in\{2 l-24,2 l-22, \ldots, 2 l-16\}$ as the last 5 roots in $\mathcal{S}$. We have $S\left(i, D_{1}\right) \cap V_{e}=$ $\{i, i+10\}$ and $S\left(i, D_{2}\right) \cap V_{e}=\{i, i+16\}$ by the previous claim.

Take the incidence vectors $\chi^{S\left(i, D_{1}\right)}$ for $i \in\{2,4, \ldots, 2 l-10\}$ as the first $l-5$ rows and $\chi^{S\left(i, D_{2}\right)}$ for $i \in\{2 l-24, \ldots, 2 l-16\}$ as the last 5 rows of $\left(A_{21} \mid A_{22}\right)$. By construction, $A_{22}$ is the $l \times l$-matrix shown in Figure 6 ( 0 -entries are dropped and the columns represent the vertices in $V_{e}$ ).

$A_{22}$ has only 1-entries on the main diagonal (coming from the first vertices in $V_{e}$ of $S\left(i, D_{1}\right)$ for $i \in\{2,4, \ldots, 2 l-10\}$ and from the second vertices in $V_{e}$ of $S\left(i, D_{2}\right)$ for $i \in\{2 l-24, \ldots, 2 l-16\})$. The only non-zero entries of $A_{22}$ below the main diagonal come from the first vertices in $V_{e}$ of $S\left(i, D_{2}\right)$ for $i \in\{2 l-24, \ldots, 2 l-16\}$. Hence, $A_{22}$ has the form

$$
A_{22}=\left(\begin{array}{c|c}
A_{22}^{\prime} & \\
\hline 0 & A_{22}^{\prime \prime}
\end{array}\right)
$$

where both matrices $A_{22}^{\prime}$ and $A_{22}^{\prime \prime}$ are invertible due to the following reasons. $A_{22}^{\prime}$ is an $(l-13) \times(l-13)$-matrix having 1 -entries on the main diagonal and 0 -entries below the main diagonal by construction; hence $A_{22}^{\prime}$ is clearly invertible. $A_{22}^{\prime \prime}$ is the $(2,13)$-circulant matrix and, therefore, clearly invertible as well. (Note that $l=13$ implies $A_{22}=A_{22}^{\prime \prime}$.)

This completes the proof that $A_{22}$ is invertible for every $l \geq 13$ with $l=1(\bmod 3)$ if we choose the set $\mathcal{S}$ of $l$ roots of (4) as constructed above.

Remark. Note that there are no mixed roots of (4) in the case $l=7$ (since $\alpha\left(W_{7}^{2}\right)=$ $2=\alpha\left(W_{14}^{4}\right)$ implies that we cannot built stable sets of size $>\alpha\left(W_{7}^{2}\right)$ in $\left.W_{14}^{4}\right)$. In the case $l=10$, there are only 5 mixed roots of size $>\alpha\left(W_{10}^{2}\right)$, namely $S\left(i, D_{1}\right)$ with $i \in\{2,4,6,8,10\}$ because of $S\left(i, D_{1}\right)=S\left(i+10, D_{1}\right)$; the sets $S\left(i, D_{2}\right)$ can be constructed only if $l \geq 13$. Hence, (4) is neither a facet of $\operatorname{STAB}\left(W_{14}^{4}\right)$ nor of $\operatorname{STAB}\left(W_{20}^{2}\right)$. Moreover, in the case $l=13$, we would obtain the same set $\mathcal{S}$ by choosing the roots $S\left(i, D_{1}\right)$ with $i \in\{2 l-8, \ldots, 2 l\}$ instead of $S\left(i, D_{2}\right)$ with $i \in\{2 l-24, \ldots, 2 l-16\}$.

Hence, we have shown that, for every $l \geq 13$ with $l=1(\bmod 3)$, there are $2 l$ roots of (4) whose incidence vectors are linearly independent, completeing the proof of Theorem 5.

\section{Proof of Theorem 6}

The aim of this section is to prove that the clique family inequality $(\mathcal{Q}, k)$ associated with any $(k-1,1)$-regular subweb $W_{l(k-1)}^{k-1}$ induces the facet (5) of $\operatorname{STAB}\left(W_{l k}^{k}\right)$ for every odd $k \geq 5$ whenever $l \geq 3 k+2$. 
For that, let $l=l^{\prime} k+2$ with $l^{\prime} \geq 3$ and $n=l k=l^{\prime} k^{2}+2 k$. Denote the set of vertices of $W_{n}^{k}$ by $V=\{1, \ldots, n\}$ and the subset of all vertices $i \in V$ with $i \nmid k$ by $V^{\prime}$, i.e., let $V^{\prime}=V \backslash\{k, 2 k, \ldots, l k\}$ be the vertex set of $W_{l(k-1)}^{k-1}$. Then $\mathcal{Q}=\left\{Q_{i}: i \in V^{\prime}\right\}$ obviously implies $I(\mathcal{Q}, k)=V^{\prime}$. As

$$
\alpha\left(W_{l(k-1)}^{k-1}\right)=\left\lfloor\frac{(k-1) l}{k}\right\rfloor=\left\lfloor l-l^{\prime}-\frac{2}{k}\right\rfloor=l-l^{\prime}-1=l^{\prime}(k-1)+1
$$

holds, the clique family inequality $(\mathcal{Q}, k)$ reads

$$
2 \sum_{i \in V^{\prime}} x_{i}+\sum_{i \in V \backslash V^{\prime}} x_{i} \leq 2 l^{\prime}(k-1)+2
$$

and is supposed to define a facet of $\operatorname{STAB}\left(W_{l k}^{k}\right)$ for any odd $k \geq 5$ if $l=l^{\prime} k+2$ and $l^{\prime} \geq 3$.

In order to verify that we have to present $l k$ roots, i.e., stable sets satisfying $(\mathcal{Q}, k)$ at equality, whose incidence vectors are linearly independent.

The maximum stable sets of $W_{l(k-1)}^{k-1}$ are independent by Trotter [16] as $k \backslash l(k-1)$ by $l=2(\bmod k)$ and $k$ odd.

This provides us already $l(k-1)$ independent roots of $(\mathcal{Q}, k)$ containing vertices from $V^{\prime}$ only. We are going to build $l$ further mixed roots containing vertices from $V^{\prime}$ as well as from $V \backslash V^{\prime}$ such that their incidence vectors are linearly independent, too.

For any vertex $i \in V \backslash V^{\prime}$, denote by $D_{i}=\{i, i+1, \ldots, i+k-1\}$ resp. $B_{i}=$ $\left\{i, i+1, \ldots, i+k^{2}-1\right\}$ the subset of $V$ consisting of $k$ resp. $k^{2}$ consecutive vertices starting in vertex $i$ (with arithmetics performed modulo $n$ ). Furthermore, define $S\left(B_{i}\right) \subset B_{i}$ by

$$
S\left(B_{i}\right)=\{i+1+j(k+1): 0 \leq j \leq k-2\}
$$

as the black vertices in Figure 7 (the squares stand for nodes in $V \backslash V^{\prime}$ ). By construction, $S\left(B_{i}\right)$ is obviously a stable set of $W_{l k}^{k}$ consisting of nodes from $V^{\prime}$ only.

Fig. 7. The stable set $S\left(B_{i}\right) \subset B_{i}$ in the case $k=5$

To build the mixed roots, we are going to use two types of partitions of $V=\{1, \ldots, n\}$ into 2 subsets $D_{j}$ of size $k$ and $l^{\prime}$ subsets $B_{i}$ of size $k^{2}$ (recall that $n=2 k+l^{\prime} k^{2}$ holds). We pick the first vertex from each subset $D_{j}$ and $S\left(B_{i}\right)$ from the involved subsets $B_{i}$ and show that the so constructed vertex sets form roots of $(\mathcal{Q}, k)$.

For every $1 \leq i \leq l$, the vertex $k i$ belongs to $V \backslash V^{\prime}$ and

$$
D_{k i} \cup B_{k(i+1)} \cup D_{k(i+1+k)} \cup \bigcup_{j=1, \ldots, l^{\prime}-1} B_{k(i+2+j k)}
$$

forms a partition of $V=\{1, \ldots, n\}$, as $k\left(i+2+\left(l^{\prime}-1\right) k\right)+k^{2}-1=k i-1(\bmod n)$ holds. Let

$$
S_{k i}=\{k i\} \cup S\left(B_{k(i+1)}\right) \cup\{k(i+1+k)\} \cup \bigcup_{j=1, \ldots, l^{\prime}-1} S\left(B_{k(i+2+j k)}\right)
$$


be the set consisting of the first vertices from $D_{k i}$ and $D_{k(i+1+k)}$ and the stable sets $S\left(B_{j}\right)$ for the involved subsets $B_{j}$.

Furthermore, for every $1 \leq i \leq l$,

$$
D_{k i} \cup \bigcup_{j=0, \ldots, l^{\prime}-3} B_{k(i+1+j k)} \cup D_{k\left(i+1+\left(l^{\prime}-2\right) k\right)} \cup B_{k\left(i+2+\left(l^{\prime}-2\right) k\right)} \cup B_{k\left(i+2+\left(l^{\prime}-1\right) k\right)}
$$

forms a second type of partition of $V=\{1, \ldots, n\}$, as $k\left(i+2+\left(l^{\prime}-2\right) k\right)+k^{2}-1=k i-1$ $(\bmod n)$. Let

$$
S_{k i}^{\prime}=\{k i\} \cup \bigcup_{j=0, \ldots, l^{\prime}-3} S\left(B_{k(i+1+j k)}\right) \cup\left\{k\left(i+1+\left(l^{\prime}-2\right) k\right)\right\} \cup \bigcup_{j=l^{\prime}-2, l^{\prime}-1} S\left(B_{k(i+2+j k)}\right)
$$

be the corresponding set consisting of the first vertices from $D_{k i}$ and $D_{k\left(i+1+\left(l^{\prime}-2\right) k\right)}$ and the stable sets $S\left(B_{j}\right)$ for the involved subsets $B_{j}$. (Note that $S_{k i}=S_{k i}^{\prime}$ iff $l^{\prime}=3$ ). We call $k i$ the start vertex of $S_{k i}$ resp. of $S_{k i}^{\prime}$.

Claim. The sets $S_{k i}$ and $S_{k i}^{\prime}$ are mixed roots of $(\mathcal{Q}, k)$ for $1 \leq i \leq l$.

Proof. By construction, we have $k i, k(i+1+k) \in S_{k i}$ and $k i, k\left(i+1+\left(l^{\prime}-2\right) k\right) \in S_{k i}^{\prime}$ (these vertices belong obviously to $V \backslash V^{\prime}$ ). The remaining vertices of $S_{k i}$ and $S_{k i}^{\prime}$ come from the sets $S\left(B_{j}\right)$ for each of the $l^{\prime}$ subsets $B_{j}$. Since $S\left(B_{j}\right)$ contains only $k-1$ vertices from $V^{\prime}$ by construction, we obtain $\left|S_{k i} \cap V^{\prime}\right|=\left|S_{k i}^{\prime} \cap V^{\prime}\right|=l^{\prime}(k-1)$ and $\left|S_{k i} \backslash V^{\prime}\right|=$ $\left|S_{k i}^{\prime} \backslash V^{\prime}\right|=2$. Thus $S_{k i}$ as well as $S_{k i}^{\prime}$ satisfy $(\mathcal{Q}, k)$ at equality for every $1 \leq i \leq l$.

It is left to show that $S_{k i}$ and $S_{k i}^{\prime}$ are stable. For that, recall first that $S\left(B_{j}\right)$ is a stable set for any $j$. Second, the last $k$ vertices of $B_{j}$ do not belong to $S\left(B_{j}\right)$ by construction (see Figure 7), thus $B_{j}$ can be followed by any subset without introducing adjacencies in $S_{k i}$ or $S_{k i}^{\prime}$. Finally, consider a subset $D_{k j}$ followed by $B_{k(j+1)}$. By construction, the last $k-1$ vertices of $D_{k j}$ as well as the first vertex of $B_{k(j+1)}$ do not belong to $S_{k i}$ or $S_{k i}^{\prime}$, thus no adjacencies are introduced again.

This implies that $S_{k i}$ and $S_{k i}^{\prime}$ are stable sets satisfying $(\mathcal{Q}, k)$ at equality.

We are now prepared to select a set of $l k$ independent roots of $(\mathcal{Q}, k)$ :

Claim. There are $l k$ roots of $(\mathcal{Q}, k)$ whose incidence vectors are linearly independent: the $l(k-1)$ maximum stable sets of $W_{l(k-1)}^{k-1}$ and the $l$ stable sets $S_{k i}$ for $1 \leq i \leq l-(k+1)$ resp. $S_{k i}^{\prime}$ for $l-k \leq i \leq l$.

Proof. The maximum stable sets of $W_{l(k-1)}^{k-1}$ are linearly independent as mentioned above. Moreover, they contain only vertices from $V^{\prime}$ whereas the stable sets $S_{k i}$ and $S_{k i}^{\prime}$ contain vertices from $V^{\prime}$ as well as vertices from $V \backslash V^{\prime}$. Thus, we are done if we can show that the incidence vectors of $S_{k i}$ for $1 \leq i \leq l-(k+1)$ and $S_{k i}^{\prime}$ for $l-k \leq i \leq l$ are linearly independent.

We construct an $(l \times l k)$-matrix $M$ having the incidence vectors of $S_{k}, \ldots, S_{k(l-(k+1))}$ as first $l-(k+1)$ rows and the incidence vectors of $S_{k(l-k)}^{\prime}, \ldots, S_{k l}^{\prime}$ as last $k+1$ rows. We show that the $(l \times l)$-submatrix $M^{\prime}$ of $M$ containing all columns corresponding to the vertices in $V \backslash V^{\prime}$ is invertible. For that, choose an ordering of the columns of $M$ s.t. the first $l$ columns correspond to the vertices $k, \ldots, l k$ in $V \backslash V^{\prime}$ and the remaining $l(k-1)$ columns correspond to the vertices in $V^{\prime}$ (see Figure 5).

Each row of $M^{\prime}$ has a 1-entry on the main diagonal (since $k i$ is the start vertex of $S_{k i}$ as well as of $S_{k i}^{\prime}$ by construction), thus we have to discuss the second 1-entries of the rows coming from the vertices $k(i+1+k) \in S_{k i}$ resp. $k\left(i+1+\left(l^{\prime}-2\right) k\right) \in S_{k i}^{\prime}$ (see Figure 5).

Let $l=l-(3 k+1)$ (we have $l \geq 1$ since $l=l^{\prime} k+2$ and $l^{\prime} \geq 3$ ). We show that $k l$ is the first column with a 1 -entry below the main diagonal, namely in the row corresponding to $S_{k(l-k)}^{\prime}$. 


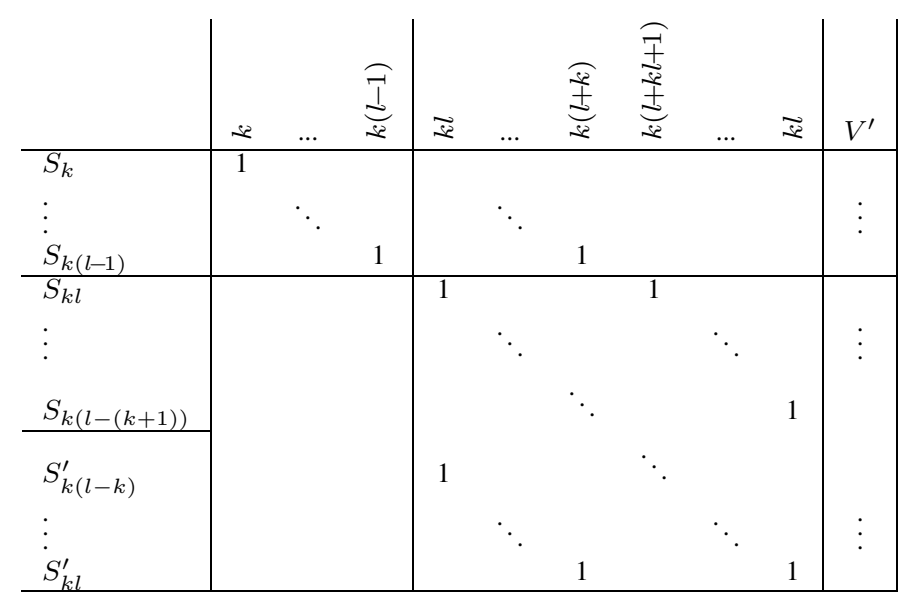

Fig. 8. The $(l \times l k)$-matrix $M$

The first $l-(k+1)$ rows of $M^{\prime}$ do not have any 1-entry below the main diagonal, since the second vertex of $S_{k j}$ in $V \backslash V^{\prime}$ is $k(j+1+k)$ and $k(j+1+k) \leq k l$ holds due to $j \leq l-(k+1)$. (In fact, the row corresponding to $S_{k(l-(k+1))}$ has its 1-entries in the columns $k(l-(k+1))$ and $k l$.

Consider now the row $l-k$ corresponding to $S_{k(l-k)}^{\prime}$. We have $S_{k(l-k)}^{\prime} \backslash V^{\prime}=\{k(l-$ $\left.k), k\left(l-k+1+\left(l^{\prime}-2\right) k\right)\right\}$ where

$$
k\left(l-k+1+\left(l^{\prime}-2\right) k\right)=k\left(-3 k+1+l^{\prime} k\right) \bmod n=k l
$$

as $l=l^{\prime} k+2$. Hence the row given by $S_{k(l-k)}^{\prime}$ has indeed a 1-entry at column $k l$.

The matrix $M^{\prime}$ is invertible, if its $(3 k+2) \times(3 k+2)$-submatrix $M^{\prime \prime}$ consisting of the colums $k l, \ldots, k l$ of the rows corresponding to $S_{k l}, \ldots, S_{k(l-(k+1))}, S_{k(l-k)}^{\prime}, \ldots, S_{k l}^{\prime}$ is invertible (since the previous part of $M^{\prime}$ has 0 -entries below the main diagonal only).

We complete the proof of this claim by showing that $M^{\prime \prime}$ is a $(3 k+2,2)$-circulant matrix: The 1-entries below the main diagonal start in column $k l$, as seen above, and end in the last row in column $k(l-(2 k+1))$ since

$$
k\left(l+1+\left(l^{\prime}-2\right) k\right)=k l+k\left(l^{\prime} k+2-(2 k+1)\right) \bmod n=k(l-(2 k+1))
$$

holds as $l=l^{\prime} k+2$, whereas the 1 -entries above the main diagonal start in column $k(l-2 k)$ due to

$$
k(l+1+k)=k(l-(3 k+1)+1+k)=k(l-2 k)
$$

and end with $k l$ in the row corresponding to $S_{k(l-(k+1))}$ as shown above. This implies that $M^{\prime \prime}$ is a $(3 k+2,2)$-circulant matrix and, therefore, invertible as $k$ is odd by our hypothesis. This complets the proof that the choosen stable sets are $l k$ independent roots of $(\mathcal{Q}, k)$.

Hence $(\mathcal{Q}, k)$ is, for any odd $k \geq 5$, a proper weak non-rank facet (5) of $\operatorname{STAB}\left(W_{k l}^{k}\right)$ if $l \geq 3 k+2$ completing the proof of Theorem 6 .

\section{Concluding remarks and open problems}

In this paper, we presented infinite sequences of not rank-perfect webs $W_{n}^{k}$ for $k=4$ (Theorem 5), all even $k \geq 6$ (Theorem 4), and all odd $k \geq 5$ (Theorem 6). Before, the case $k=3$ was settled in [14]. Applying the construction from [15] yields that there are only finitely many rank-perfect webs $W_{n}^{k}$ for all values of $k \geq 3$ (Corollary 4), implying that almost all webs with fixed clique number at least 4 are not rank-perfect (Corollary 5). 
For our construction, we used clique family inequalities associated with certain subwebs yielding 1/2-valued facets; the construction from [15] does not change the involved coefficients and, therefore, the stable set polytopes of almost all webs admit 1/2-valued facets.

According to Ben Rebea's Conjecture [12], the stable set polytopes of quasi-line graphs (and therefore of webs) have clique family inequalities as only non-trivial facets. However, clique family inequalities constitute a large class of valid inequalities; even verifying Ben Rebea's Conjecture would provide no information about which inequalities are essential among them. The following conjecture addresses this problem for the subclass of webs.

Conjecture 1. Every facet of $\operatorname{STAB}\left(W_{n}^{k}\right)$ belongs to one of the following classes:

(0) nonnegativity constraints,

(i) clique constraints,

(ii) full rank constraint,

(iii) clique family inequalities $\left(\mathcal{Q}, k^{\prime}+1\right)$ associated with proper subwebs $W_{n^{\prime}}^{k^{\prime}}$ where $\left(k^{\prime}+1\right) \nmid n^{\prime}$ and $\alpha\left(W_{n^{\prime}}^{k^{\prime}}\right)<\alpha\left(W_{n}^{k}\right)$.

All non-rank facets known so far for webs are of type (iii). Note that a web $W_{n}^{k}$ usually has subwebs $W_{n^{\prime}}^{k^{\prime}}$ for all values $1 \leq k^{\prime}<k$. Hence, we expect that the stable set polytope of $W_{n}^{k}$ admits $(k-2) /(k-1)$-valued facets. The conjecture implies in particular, that the stable set polytopes of all webs $W_{n}^{3}$ have 1/2-valued facets only, where for all webs $W_{n}^{k}$ with $k>3$ larger coefficients are required. In fact, Liebling et al. [11] proved recently that, for any odd $k \geq 5$, the stable set polytope of $W_{k^{2}}^{k}$ has an $(k-2) /(k-1)$-valued facet. Hence, further effort is needed for having a complete description of stable set polytope of webs (and for the larger class of fuzzy circular interval graphs).

Acknowledgements. The first author was supported by DONET/ZIB and the second author was supported by the Deutsche Forschungsgemeinschaft (Gr 883/9-1).

\section{References}

1. M. Chudnovsky, N. Robertson, P. Seymour, and R. Thomas, Progress on Perfect Graphs. Mathematical Programming B 97 (2003) 405-422

2. M. Chudnovsky and P. Seymour, Claw-free graphs VI. The structure of quasi-line graphs. manuscript (2004).

3. V. Chvátal, On Certain Polytopes Associated with Graphs. J. Combin. Theory B 18 (1975) 138-154

4. V. Chvátal, On the Strong Perfect Graph Conjecture. J. Combin. Theory B 20 (1976) 139-141

5. G. Dahl, Stable Set Polytopes for a Class of Circulant Graphs. SIAM J. Optim. 9 (1999) 493-503

6. J.R. Edmonds and W.R. Pulleyblank, Facets of 1-Matching Polyhedra. In: C. Berge and D.R. Chuadhuri (eds.) Hypergraph Seminar. Springer-Verlag, Heidelberg, (1974) 214-242

7. A. Galluccio and A. Sassano, The Rank Facets of the Stable Set Polytope for Claw-Free Graphs. J. Comb. Theory B 69 (1997) 1-38

8. G. Giles and L.E. Trotter, jr., On Stable Set Polyhedra for $K_{1,3}$-free Graphs. J. Comb. Theory B 31 (1981) 313-326

9. M. Grötschel, L. Lovász, and A. Schrijver, Geometric Algorithms and Combinatorial Optimization. SpringerVerlag, (1988)

10. J. Kind, Mobilitätsmodelle für zellulare Mobilfunknetze: Produktformen und Blockierung. PhD thesis, RWTH Aachen (2000)

11. T.M. Liebling, G. Oriolo, B. Spille, and G. Stauffer, On Non-Rank Facets of the Stable Set Polytope of ClawFree Graphs and Circulant Graphs. Submitted to Math. Methods of Operations Research

12. G. Oriolo, Clique Family Inequalities for the Stable Set Polytope for Quasi-Line Graphs. In: Special Issue on Stability Problems, Discrete Applied Math. 132 (2003) 185-201

13. M.W. Padberg, Perfect Zero-One Matrices. Math. Programming 6 (1974) 180-196

14. A. Pêcher and A. Wagler, On Non-Rank Facets of Stable Set Polytopes of Webs with Clique Number Four, ZIB-Report ZR 03-01 (2003)

15. A. Pêcher and A. Wagler, A construction for non-rank facets of stable set polytopes of webs, LaBRI-Report RR-1304-03 (2003)

16. L.E. Trotter, jr., A Class of Facet Producing Graphs for Vertex Packing Polyhedra. Discrete Math. 12 (1975) 373-388

17. A. Wagler, Antiwebs are rank-perfect. to appear in: Quarterly Journal of the Belgian, French and Italian OR Societies 